\title{
Facilitação neuromuscular proprioceptiva em pacientes com acidente cerebrovascular
}

\author{
Neuromuscular proprioceptive facilitation in in patients with stroke
}

\section{Facilitación neuromuscular propioceptiva en pacientes con accidente cerebrovascular}

Kaíza Kelly Sousa dos Santos ${ }^{1}$, Tassiane Maria Alves Pereira², Maria Claudilene de Andrade Ramos ${ }^{3}$, Sergio Augusto Nader Damasceno ${ }^{4}$, Jordano Leite Cavalcante Macêdo ${ }^{5}$, Marco Azizi ${ }^{6}$, Adalgiza Mafra Moreno ${ }^{6}$, Marco Orsini ${ }^{6,7}$, Carlos Henrique Melo Reis ${ }^{6}$, Janaína De Moraes Silva $7,8,9$, Victor Hugo Do Vale Bastos ${ }^{9,10}$

1.Centro Universitário Santo Agostinho (UNIFSA), Teresina-PI, Brasil.

2.Pós-graduanda em Fisioterapia Hospitalar (INSPIRAR), Teresina-PI, Brasil.

3.Faculdade Maurício de Nassau (UNINASSAU), Teresina-PI, Brasil.

4.Médico, Especialista em Anatomia Humana, Mestre em Educação (UNIG -RJ), Professor de Anatomia Humana nas graduações em Medicina, Odontologia e Nutrição (UNIG-RJ) e colaborador do LAMCEF (UFDPAR -PI). Rio de Janeiro-RJ, Brasil.

5. Mestre em Engenharia Biomédica, Universidade do Vale do Paraíba, UNIVAP, São José dos Campos-SP, Brasil.

6.Universidade Iguaçu (UNIG- RJ), Nova Iguaçu-RJ; Docente do Mestrado Multiprofissional em Ciências Aplicadas em Saúde. Rio de Janeiro-RJ, Brasil.

7.Pós-Doutorando em Ciências Biomédicas - UFDPAR/UFPI, Parnaíba-PI, Brasil.

8.Universidade Estadual do Piauí (UESPI), Teresina-PI, Brasil.

9.Laboratório de Mapeamento Cerebral e Funcionalidade (UFPI-CMRV-LAMCEF), Parnaíba-PI, Brasil.

10. Estágio Pós Doutoral, PPG Neurologia/Neurociências, UFF/RJ, Rio de Janeiro-RJ, Brasil.

\section{Resumo}

Introdução. O Acidente Cerebrovascular (AVC) pode ser definido como um dano ocasionado por distúrbios hemodinâmicos e da coagulação, ainda que não apresente mudanças detectáveis nas artérias ou veias de grande relevância médica. Diante disso, a Facilitação Neuromuscular Proprioceptiva (FNP) é definida como um conceito de promoção ou aceleração do mecanismo neuromuscular através de estímulos de receptores sensitivos. Método. Tratase de uma revisão de literatura operacionalizada por meio de seis etapas as quais estão estreitamente interligadas: elaboração da pergunta norteadora, busca na literatura, coleta de dados, análise crítica dos estudos incluídos, discussão dos resultados e apresentação da revisão. Resultados. Os resultados apresentaram um total de quatro estudos de diferentes países, sendo dois randomizados controlados, um randomizado e um não randomizado. Conclusão. Concluiu-se a relevância da cinesioterapia utilizando o FNP no tratamento das sequelas geradas pelo AVC não só como maneira alternativa de intervenção, mas como fator determinante na redução do tempo da permanência dos pacientes nos serviços de saúde e consequentemente da redução de gastos no sistema, bem como incrementador na qualidade de vida dos pacientes.

Unitermos. Doença; Acidente Cerebrovascular; Facilitação Neuromuscular Proprioceptiva; Fisioterapia

\footnotetext{
Abstract

Introduction. Stroke can be defined as a damage caused by hemodynamic and coagulation disorders, although it does not present detectable changes in arteries or veins of great medical relevance. In view of this, Proprioceptive Neuromuscular Facilitation (PNF) is defined as a concept of promoting or accelerating the neuromuscular mechanism through stimuli from sensitive receptors. Method. This is a literature review made operational through six steps which are closely interconnected: elaboration of the guiding question, literature search, data collection, critical analysis of the included studies, discussion of the results and presentation of the review. Results. The results presented a total of 4 studies from different countries, two randomized controlled, one randomized and one nonrandomized. Conclusion. The relevance of kinesiotherapy using FNP in the treatment of sequelae generated by the stroke was concluded not only as an alternative way of intervention, but also as a determining factor in reducing the length of stay of patients in health services and consequently reducing costs in the system, as well as increasing the quality of life of patients.

Keywords Disease; Stroke; Facilitation; Neuromuscular; Proprioceptive; Physiotherapy
} 


\section{Resumen}

Introducción. El accidente cerebrovascular (ACV) puede definirse como un daño causado por trastornos hemodinámicos y de coagulación, aunque no presenta cambios detectables en arterias o venas de gran relevancia médica. En vista de esto, la facilitación neuromuscular propioceptiva (PNF) se define como un concepto de promoción o aceleración del mecanismo neuromuscular a través de estímulos de receptores sensibles. Método. Esta es una revisión de la literatura que se hizo operativa a través de seis pasos estrechamente interconectados: elaboración de la pregunta guía, búsqueda de literatura, recolección de datos, análisis crítico de los estudios incluidos, discusión de los resultados y presentación de la revisión. Resultados. Los resultados presentaron un total de 4 estudios de diferentes países, dos controlados aleatorios, uno aleatorio y uno no aleatorio. Conclusion. La relevancia de la kinesioterapia con FNP en el tratamiento de las secuelas generadas por el accidente cerebrovascular se concluyó no solo como una forma alternativa de intervención, sino también como un factor determinante para reducir la duración de la estancia de los pacientes en los servicios de salud $y$, en consecuencia, reducir los costos en el sistema, además de aumentar la calidad de vida de los pacientes.

Palabras clave. enfermedad; Accidente cerebrovascular; Facilitación neuromuscular propioceptiva; Fisioterapia

Trabalho realizado na Universidade Estadual do Piauí (UESPI), Teresina-PI, Brasil.

\section{INTRODUÇÃO}

O Acidente Cerebrovascular (AVC) ocorre devido a danos ocasionados por distúrbios hemodinâmicos e da coagulação, podendo levar a comprometimentos neurológicos e a incapacidade ou morte. Tendo sua origem isquêmica ou hemorrágica, a primeira ocorre através da obstrução vascular localizada, cessando o fornecimento de oxigênio e glicose ao cérebro, isso afeta os processos metabólicos da região envolvida. Ao passo que 0 hemorrágico tem como fator causador um aneurisma ou trauma nas áreas extravasculares do cérebro ${ }^{1}$.

Estudos revelam que entre as pessoas acometidas por AVC, cerca de 37\% evoluem com manifestações discretas, $16 \%$ são qualificadas como moderada limitação funcional e 
32\% apresentam alteração intensa ou grave da funcionalidade, tornando-se cadeirantes ou limitados ao leito, somente $15 \%$ dos acometidos permanece com a capacidade funcional preservada ${ }^{2}$. Com relação à incidência trata-se de uma doença que acomete predominantemente adultos de meia idade, idosos, negros e homens, onde 85\% dos casos possui origem isquêmica e estão ligados a fatores de riscos como a hipertensão arterial, diabetes mellitus, tabagismo, doenças cardíacas, hipercolesterolimia, sedentarismo, estresse, obesidade e predisposição genética ${ }^{1}$.

A intervenção fisioterapêutica voltada para os pacientes com disfunções ocasionadas pelo AVC, tem basicamente dois objetivos. O primeiro, adequar o tônus no domínio afetado, e o segundo, fortalecer os músculos espásticos e seus antagonistas, promovendo melhora funcional aos pacientes, beneficiando assim, melhor independência3,4.

O tratamento fisioterapêutico visa auxiliar cada paciente a atingir o mais alto nível funcional possível. Diante disso, a Facilitação Neuromuscular Proprioceptiva (FNP) é definida como um conceito gera o movimento funcional por meio da facilitação, inibição, fortalecimento e relaxamento de grupos musculares. As técnicas se valem de contrações musculares concêntricas, excêntricas e isométricas, juntamente com a aplicação de uma resistência gradual e procedimentos facilitatórios adequados, todos adaptados para alcançar as necessidades de cada paciente. Esses procedimentos promovem um aumento da habilidade do 
paciente em se mover e se manter estável, orientam o movimento utilizando contatos manuais e resistência apropriados, fazem com que o paciente obtenha coordenação motora e sincronismo, ampliam a capacidade de resistência do indivíduo e evitam a fadiga 4 .

Os níveis de comprometimento funcional decorrentes do AVC variam de um paciente para o outro e a execução de habilidades como, por exemplo, vestir-se, comer, tomar banho sozinho e caminhar pequenas distâncias de forma independente podem ser muito prejudicadas, levando o indivíduo a um quadro de incapacidade funcional ${ }^{3}$. Também são prejudicadas as Atividades de Vida Diária (AVD), que se referem às atividades um pouco mais complexas do cotidiano, como por exemplo, passear, fazer compras, limpar a casa, lavar roupa, dirigir, utilizar meios de transporte coletivo entre outros ${ }^{5}$.

A FNP além de um conjunto de técnicas muito utilizado na prática clínica promove o desempenho dos pacientes durante atividades de vida diária e contribui, assim, na melhora da qualidade de vida. Dessa forma, a presente revisão tem por objetivo descrever a os efeitos das técnicas da FNP na melhora da qualidade de vida de pacientes acometidos pelo AVC.

\section{MÉTODO}

Foi realizada uma revisão sistemática de literatura pautada na: 1) busca nas bases de dados; 2) definição de 
critérios de inclusão e exclusão; e 3) avaliação da qualidade metodológica das produções.

Uma busca sistemática nas bases de dados PEDRO, MEDLINE, COCHRANE, SCIELO e CENTRAL por artigos publicados entre os anos de 2014 a 2019. As palavras-chave utilizadas para a busca seguiram a descrição dos termos Mesh, sendo elas: 'AVC', 'AVE', 'FNP', 'Proprioceptive Neuromuscular Facilitation', 'Neuromuscular', 'Neuromuscular Facilitation', 'Life Quality', 'Stroke', 'Physical Therapist Assistants', 'Physical Therapists', 'Physical Therapy Specialty', 'Physical Therapy Modalities'. A busca também foi realizada com os descritores em língua portuguesa e espanhola. As palavras-chave foram combinadas utilizandose os operadores booleanos OR e AND, sem restrição linguística.

Para serem incluídos na presente revisão sistemática, os estudos identificados pela estratégia de busca deveriam consistir em ensaios clínicos randomizados ou quaserandomizados que utilizassem a intervenção fisioterapêutica com facilitação neuromuscular proprioceptiva como forma de tratamento para as sequelas de AVC e a comparassem a um grupo controle sem intervenção fisioterapêutica ou a um grupo placebo. Foram excluídos estudos que tratavam o AVC com métodos farmacológicos ou que utilizassem estimulação elétrica.

Os resumos dos estudos identificados pela busca foram avaliados segundo os critérios de elegibilidade acima citados, e aqueles que geraram dúvidas foram retidos para uma 
posterior avaliação do texto na íntegra. Os selecionados para inclusão tiveram sua qualidade metodológica avaliada.

A escala de qualidade metodológica PEDro foi utilizada para avaliar a qualidade dos estudos individuais incluídos nesta revisão. A escala PEDro foi projetada para ajudar os usuários do banco de dados a identificar rapidamente quais dos ensaios clínicos randomizados controlados. Dois revisores avaliaram independentemente a qualidade metodológica de cada estudo. Quaisquer diferenças nas pontuações dos revisores independentes foram avaliadas, discutidas e resgatadas, resultando em uma pontuação geral para a qualidade metodológica. Portanto, cada artigo recebeu uma pontuação entre 0 e 10. Estudos de alta pontuação usaram um desenho de pesquisa de estudo controlado randomizado. Estudos randomizados com um grupo experimental e controle têm mais poder estatístico do que estudos com apenas um grupo experimental. Algumas limitações da literatura nessa área incluem amostras pequenas, homogeneidade da amostra, cegamento deficiente (de sujeitos, prestadores de tratamento e avaliadores) e nenhuma ou limitada randomização.

\section{RESULTADOS}

De 493 artigos inicialmente identificados por meio das bases de dados pesquisadas, 12 foram retirados para uma avaliação criteriosa, sendo quatro deles excluídos pela análise de resumos. Foram avaliados para elegibilidade oito 
textos completos, dos quais quatro foram excluídos por não preencherem os critérios de inclusão (Figura 1). Foram incluídos um total de quatro estudos de diferentes países, sendo dois randomizados controlados, um randomizado, um não randomizado.

Figura 1. Fluxograma de seleção dos ensaios clínicos.

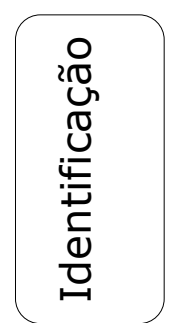

Estudos potencialmente relevantes identificados por meio da busca eletrônica nas bases de dados: MEDLINE $(n=247)$, COCHRANE $(n=74)$, PEDRO $(n=$ $6)$, SCIELO $(n=89)$ e CENTRAL $(n=77)$
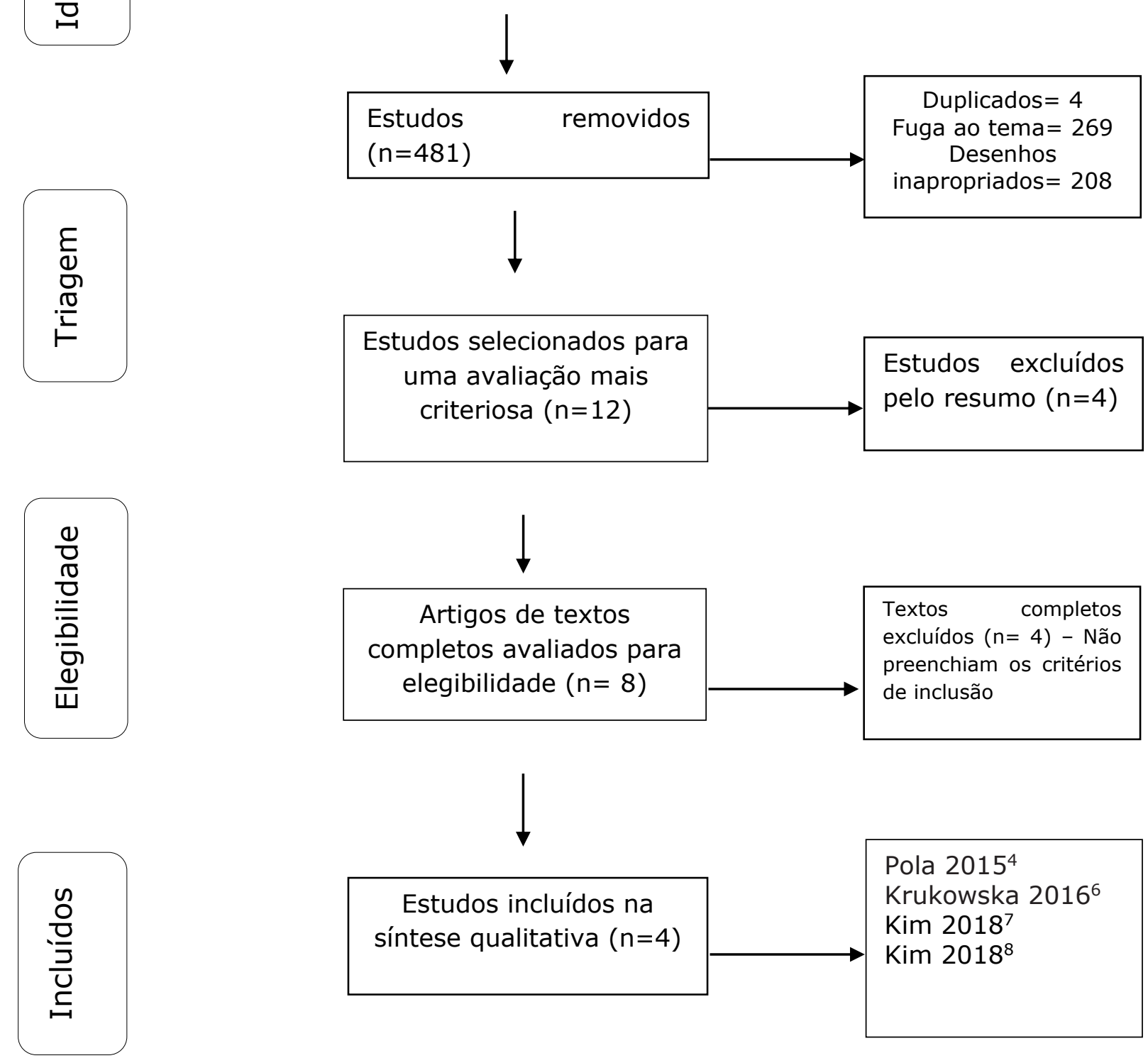
Quadro 1. Sumário dos estudos incluídos.

\begin{tabular}{|c|c|c|c|c|c|}
\hline Autor & Amostra & Grupos & Intervenção & $\begin{array}{c}\text { Variáveis } \\
\text { analisadas }\end{array}$ & Resultados \\
\hline Pola $2015^{4}$ & $\mathrm{~N}=4$ & $\begin{array}{l}\text { Grupo } \\
\text { (residentes } \\
\text { da } \\
\text { comunidade } \\
\text { local } \\
\text { selecionados } \\
\text { de forma } \\
\text { aleatória). }\end{array}$ & 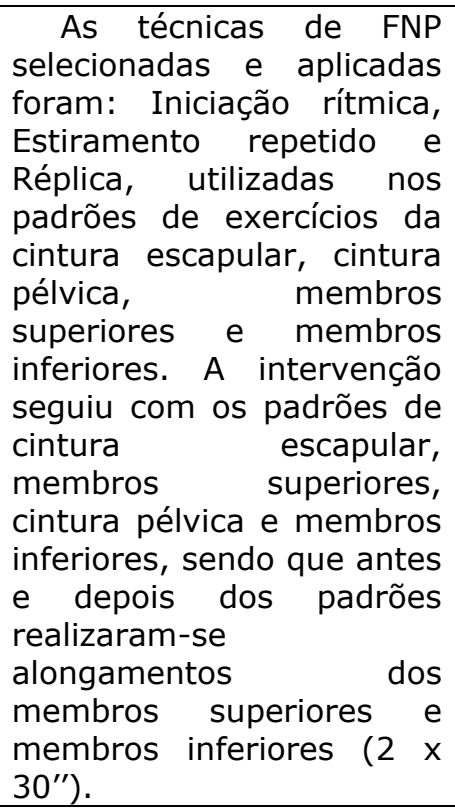 & $\begin{array}{l}\text { As variáveis } \\
\text { investigadas foram } \\
\text { a percepção do } \\
\text { nível de esforço, } \\
\text { frequência cardíaca } \\
\text { e a capacidade } \\
\text { funcional pelo } \\
\text { instrumento: } \\
\text { Escala de Borg e os } \\
\text { testes Velocidade } \\
\text { Habitual da Marcha } \\
\text { (VHM); Time Up } \\
\text { And Go (TUG); } \\
\text { Teste de } \\
\text { Caminhada de } 6 \\
\text { minutos (TC6'); } \\
\text { Velocidade de subir } \\
\text { e descer escadas. }\end{array}$ & $\begin{array}{l}\text { As respostas observadas } \\
\text { nesse estudo após o } \\
\text { programa de exercício } \\
\text { mostraram elevação do } \\
\text { desempenho nos testes } \\
\text { VHM, TUG e TC6' } \\
\text { (velocidade) em todos ou em } \\
\text { maior parte dos } \\
\text { participantes. A distância } \\
\text { percorrida no TC6' foi } \\
\text { ultrapassada somente pela } \\
\text { metade dos participantes, e } \\
\text { na velocidade de subida e } \\
\text { descida somente um } \\
\text { participante e unoluiu. } \\
\text { Observou-se redução da FC } \\
\text { inicial e final antes e depois } \\
\text { do TC6' em três } \\
\text { participantes. }\end{array}$ \\
\hline $\begin{array}{l}\text { Krukowska } \\
2016^{6}\end{array}$ & $\mathrm{~N}=72$ & $\begin{array}{l}\text { Os pacientes } \\
\text { foram } \\
\text { divididos em } \\
4 \text { grupos por } \\
\text { uma } \\
\text { randomizaçã } \\
\text { o simples. Os } \\
\text { critérios para } \\
\text { essa divisão } \\
\text { foram: o lado } \\
\text { do corpo } \\
\text { (direito ou } \\
\text { esquerdo) } \\
\text { afetado pela } \\
\text { paresia e os } \\
\text { métodos de } \\
\text { reabilitação } \\
\text { aplicados. }\end{array}$ & $\begin{array}{l}\text { Todos os pacientes foram } \\
\text { submetidos ao método } \\
\text { cinesioterápico } \\
\text { recomendado } \\
\text { (randomizado), } 35 \text { sessões } \\
\text { de terapia, todos os dias, } \\
\text { por um período de seis } \\
\text { semanas. Antes do início da } \\
\text { terapia e após } 6 \text { semanas } \\
\text { foi medida a área total do } \\
\text { suporte e o comprimento } \\
\text { do caminho (COP (Center } \\
\text { Of Pressure) mede a } \\
\text { pressão do pé) usando } \\
\text { plataforma do } \\
\text { estabilômetro - alfa. de }\end{array}$ & $\begin{array}{l}\text { Avaliar o efeito dos } \\
\text { métodos NDT- } \\
\text { Bobath e PNF no } \\
\text { suporte de campo e } \\
\text { o comprimento } \\
\text { total do trajeto } \\
\text { mede a pressão do } \\
\text { pé (COP) em } \\
\text { pacientes após } \\
\text { acidente vascular } \\
\text { cerebral. }\end{array}$ & $\begin{array}{l}\text { A maior melhoria foi obtida } \\
\text { em grupos com terapia NDT- } \\
\text { Bobath. O método NDT- } \\
\text { Bobath para melhorar o } \\
\text { equilíbrio do corpo é um } \\
\text { método mais eficaz de } \\
\text { tratamento em comparação } \\
\text { com o método PNF. Em } \\
\text { pacientes com AVC, a } \\
\text { eficácia do método NDT- } \\
\text { Bobath não depende da } \\
\text { paresia da mão. }\end{array}$ \\
\hline
\end{tabular}

Facilitação neuromuscular Proprioceptiva (FNP /PNF); Velocidade Habitual da Marcha (VHM); Time Up And Go (TUG); ; Teste de Caminhada de 6 minutos (TC6'); comprimento do caminho (COP (Center Of Pressure); NDT ( tratamento neurodesenvolvimental/ Bobath); Teste de Caminhada de 10 m (10MWT); Esteira rolante (TT) ; FNPN (low-leg taping); Facilitação neuromuscular Proprioceptiva e esteira rolante (PNFLT-TT). 
Quadro 1 (cont.). Sumário dos estudos incluídos.

\begin{tabular}{|c|c|c|c|c|c|}
\hline Kim 20187 & $\mathrm{N}=23$ & $\begin{array}{l}\text { Os pacientes } \\
\text { foram } \\
\text { aleatoriamen } \\
\text { te } \\
\text { designados } \\
\text { para um } \\
\text { grupo de } \\
\text { treinamento } \\
\text { em esteira ( } n \\
=11) \text { e um } \\
\text { grupo de } \\
\text { treinamento } \\
\text { combinado } \\
(n=12) .\end{array}$ & $\begin{array}{l}\text { O tempo de TUG } \\
\text { programado, o teste de } \\
\text { caminhada de } 10 \mathrm{~m} \\
(10 \mathrm{mWT} \text { e o teste de } \\
\text { caminhada de } 6 \text { minutos } \\
\text { (TC6) foram identificados } \\
\text { como desfechos primários. } \\
\text { Neste estudo, os } \\
\text { programas de treinamento } \\
\text { de } 40 \text { minutos para ambos } \\
\text { os grupos ocorreram } 5 \\
\text { vezes por semana durante } \\
6 \text { semanas, com } 5 \text { minutos } \\
\text { sendo alocados para o } \\
\text { aquecimento, } 30 \text { minutos } \\
\text { dedicados ao exercício } \\
\text { principal e } 5 \text { minutos para } \\
\text { o resfriamento. }\end{array}$ & $\begin{array}{lr}\text { Investigar } & \text { os } \\
\text { efeitos } & \text { do } \\
\text { treinamento } & \\
\text { combinado usando } \\
\text { padrões ra rão } \\
\text { facilitaçaro } \\
\text { neuromuscular } \\
\text { proprioceptiva } \\
\text { (PNF) e esteiras } \\
\text { sobre o equilíbrio e } \\
\text { capacidade de } \\
\text { andar de pacientes } \\
\text { com AVC }\end{array}$ & $\begin{array}{l}\text { Alterações no TUG, } 10 M W T \text { e } \\
6 M W \text { antes e após as } \\
\text { intervenções foram } \\
\text { significativamente diferentes } \\
\text { para o grupo experimental e } \\
\text { o grupo controle }(p<0,05) \text {. }\end{array}$ \\
\hline Kim $2018^{8}$ & $\mathrm{~N}=27$ & $\begin{array}{l}\text { Um grupo } \\
\text { controle } \\
\text { alocado por } \\
\text { AVC }(n=13) \\
\text { e um grupo } \\
\text { experimental } \\
(n=14) .\end{array}$ & $\begin{array}{l}\text { O grupo controle realizou o } \\
\text { TT e o grupo experimental } \\
\text { realizou o TT utilizando o } \\
\text { PNF com fita adesiva cinco } \\
\text { vezes por semana durante } \\
6 \text { semanas. A marcha e a } \\
\text { capacidade de equilíbrio } \\
\text { foram mensuradas } \\
\text { utilizando-se o teste de } \\
\text { caminhada de } 6 \text { minutos } \\
\text { (TC6), o teste de } \\
\text { caminhada de } 10 \text { metros } \\
\text { (TCM) o teste de tempo } \\
\text { de ir e vim (TUG). }\end{array}$ & $\begin{array}{l}\text { O estudo teve } \\
\text { como objetivo } \\
\text { investigar o efeito } \\
\text { do treinamento em } \\
\text { esteira rolante (TT) } \\
\text { usando o FNPN } \\
\text { (low-leg taping) na } \\
\text { habilidade de andar } \\
\text { e equilíbrio em } \\
\text { pacientes com AVC }\end{array}$ & $\begin{array}{l}\text { Após a intervenção, o grupo } \\
\text { PNFLT-TT apresentou } \\
\text { alterações mais efetivas que } \\
\text { o grupo TT no TC6min, no } \\
\text { TC10min e no TUG (P <0,05 } \\
\text { e <0,01) entre o grupo } \\
\text { PNFLT-TT e TT. A } \\
\text { intervenção do PNFLT-TT foi } \\
\text { eficaz em melhorar a } \\
\text { capacidade de andar e } \\
\text { equilíbrio em pacientes com } \\
\text { AVC }\end{array}$ \\
\hline
\end{tabular}

Facilitação neuromuscular Proprioceptiva (FNP /PNF); Velocidade Habitual da Marcha (VHM); Time Up And Go (TUG); ; Teste de Caminhada de 6 minutos (TC6'); comprimento do caminho (COP (Center Of Pressure); NDT ( tratamento neurodesenvolvimental/ Bobath); Teste de Caminhada de 10 m (10MWT); Esteira rolante (TT) ; FNPN (low-leg taping); Facilitação neuromuscular Proprioceptiva e esteira rolante (PNFLT-TT).

Quanto aos aspectos da qualidade metodológica nenhum houve mascaramento. Todos fizeram análise com intenção de tratar através da facilitação neuromuscular proprioceptiva, e em todos os estudos houve sigilo de alocação adequado. As características basais dos pacientes não foram semelhantes nos estudos incluídos, bem como os detalhes metodológicos. Entretanto os procedimentos foram realizados em adultos jovens, adultos e idosos. 
Quanto aos critérios do método de intervenção manual houve heterogeneidade dos artigos selecionados. Para homogeneizar os dados, devido a divergência entre os métodos de avaliação usados nos ensaios clínicos incluídos neste estudo. Considerou se como um resultado positivo clinicamente relevante qualquer grau de alívio dos sintomas.

\section{DISCUSSÃO}

O AVC define-se como a interrupção do fluxo de sangue para o encéfalo, ocasionado geralmente por ruptura de um vaso sanguíneo ou bloqueio através de um coágulo, onde o fornecimento de oxigênio e nutrientes é impedido, gerando lesões ao tecido encefálico variando conforme a área e a extensão do tecido acometido 9 .

Epidemiologicamente, o Brasil apresenta um acentuado aumento de óbitos por doenças cerebrovasculares e também por um número maior de indivíduos com doenças e incapacidades crônicas devido ao envelhecimento populacional. Dentro das doenças cerebrovasculares, o AVC se destaca como uma das maiores preocupações da atualidade, de acordo com pesquisas, essa enfermidade tem sido a terceira maior causa de óbitos por doença no mundo. As doenças cardíacas são consideradas o segundo maior fator de risco para quadros embólicos e aterotrombóticos, assim como, a hipertensão arterial é considerada um fator de risco para o $\mathrm{AVC}^{10}$. 
A FNP é um conceito de tratamento fundamentado no princípio de que todos os seres humanos têm uma potencialidade inexplorada. Este método emprega o enfoque terapêutico positivo e uma abordagem global de tratamento tendo como objetivo facilitar a obtenção do mais alto coeficiente funcional do indivíduo. Essa metodologia de tratamento terapêutico é amplamente utilizada na prática clínica fisioterapêutica buscando melhorar a performance do sistema neuromuscular através da estimulação dos exteroceptores periféricos e proprioceptores musculares e $\operatorname{articulares}^{11}$.

A fim de se tornar funcionalmente independente, o paciente deve ser capaz de realizar as suas atividades de vida diária, e para que isso ocorra, é indispensável à adequada manutenção da ativação muscular que propicia a geração de força necessária para a execução dessas atividades básicas ${ }^{12}$. Com isso o conceito FNP norteia-se pelos mecanismos de aprendizagem motora do modelo de Classificação Internacional de Funcionalidade, Incapacidade e Saúde (CIF) em todo o processo de avaliativo e no planejamento do tratamento, juntando as questões da abordagem funcional, positiva e integrada, analisando o indivíduo como um todo e seu ambiente nas esferas física, psíquica e social ${ }^{4}$.

A FNP possui procedimentos e princípios básicos, como: contato manual, posicionamento do corpo e biomecânica, tração e aproximação, estiramento, comando verbal (estímulo verbal), visão, sincronização, padrões de 
facilitatórios, resistência e irradiação. Pelo contato manual, o terapeuta faz a estimulação dos receptores cutâneos e de pressão do indivíduo, melhorando a resposta motora através da orientação espacial do corpo em relação ao ambiente físico, promovendo a ativação muscular de estabilidade e a movimentação durante uma atividade dinâmica ${ }^{13,14}$.

No conceito FNP é utilizado o contato lumbrical, formado pela flexão das articulações metacarpofalangeanas e extensão das articulações interfalangeanas que favorecem o controle do movimento em três dimensões. O terapeuta precisa realizar o posicionamento das mãos de forma que a pressão provocada seja contrária ao sentido do movimento almejado, assim o movimento será estimulado por meio do sinergismo muscular. A pressão, provocando uma resistência externa ao músculo amplia sua capacidade de contrair ${ }^{5}$.

Para uma adequada aplicação de contatos manuais é preciso um apropriado posicionamento e mecânica corporal do fisioterapeuta, que têm de estar posicionado com seu centro gravitacional e base de apoio alinhados com 0 movimento aspirado ou com a força empregada para que haja um mais perfeito controle do movimento e da resistência. $O$ posicionamento se realiza com a coluna em disposição neutra e ombros, quadris, braços e mãos direcionados ao movimento. A resistência durante a movimentação parte do corpo do terapeuta, que se vale do peso do corpo para aplicação de uma resistência prolongada sem fadigar, ao passo que os braços e as mãos continuam posicionados de maneira confortável ${ }^{11}$. 
A tração e aproximação empregam vetores de força de alongamento ou compressão para tornar mais fácil a estabilidade o movimento. A tração é conceituada como alongamento de uma parte (tronco ou extremidade) e o afastamento das superfícies articulares. Por meio da tração é possível melhorar movimentos, ajudar no alongamento e, por fim, fornecer a resistência ${ }^{15}$.

Os padrões da FNP têm direcionamento em diagonal e espiral para promoverem uma consonância com as propriedades do sistema musculoesquelético, além de estarem dispostos de maneira alinhada com o arranjo topográfico dos músculos. Esses padrões exibem componentes de movimentos tridimensionais, associando então, o plano sagital (flexão e extensão); o plano frontal ou coronal (abdução e adução) e o plano transversal (rotações) ${ }^{11}$.

A palavra irradiação caracteriza o acontecimento pertinente à ativação de grupos musculares, de um seguimento corporal, ocasionando ativação muscular em outra região do corpo por meio das cadeias musculares. A irradiação, dentro de um programa de tratamento para o aprendizado motor, é empregada como uma medida inicial de tratamento, buscando o recrutamento muscular de seguimentos distantes que estejam lesados, fracos ou imobilizadas e que na ocasião não seja possível ser trabalhados de maneira direta, funcionando como uma preparação para fases posteriores da reabilitação ${ }^{16}$. 
O comprometimento do membro superior é um dos fatores mais importantes dentro das restrições funcionais em pacientes pós-AVC, onde a falta de utilização do lado parético pode ocasionar o fenômeno conhecido como "desuso aprendido", no qual os pacientes param de usar o membro superior afetado passando a utilizar somente o membro superior não parético ao executar suas atividades e participação na vida cotidiana. Esse fato gera mudanças impróprias em nível de neuroplasticidade, considerado como fator maléfico ao controle motor ${ }^{7,17}$.

A fisioterapia estimula a neuroplasticidade e ajuda na reabilitação funcional de pacientes neurológicos, uma vez que esta está sendo um foco atual de pesquisa em Neurologia. Funcionalmente é possível que sejam criados novos redes ou caminhos nervosos alternativos, decorrentes de brotação massiva e sinaptogênese reativa nos axônios intactos e não lesionados ${ }^{13}$.

Com isso estudos recentes como o de $\mathrm{Kim}^{8}$ têm evidenciado que o tratamento nos indivíduos portadores de sequelas de AVC por meio de técnicas fisioterapêuticas pode estimular a boa neuroplasticidade. Entre as possíveis escolhas de tratamento estão técnicas como, a eletroestimulação, exercícios cinesioterapêuticos (Kabat), para trabalhar esta patologia. 


\section{CONCLUSÃO}

Nesta revisão é possível perceber a importância da terapia manual utilizando a Facilitação Neuromuscular Proprioceptiva no tratamento das sequelas geradas pelo acidente vascular encefálico não só como maneira alternativa de intervenção, mas como fator determinante na redução do tempo da permanência dos pacientes nos serviços de saúde e consequentemente da redução de gastos no sistema, bem como incrementador na qualidade de vida dos pacientes uma vez que proporciona o retorno dos pacientes as suas atividades de vida diária em funções simples e complexas além de se tratar de técnicas de fácil aplicabilidade e reprodutibilidade.

\section{REFERÊNCIAS}

1.Gouvêa D, Gomes CSP, Melo SC, Abrahão PN, Barbieri G. Vascular Encefálico: Uma Revisão Da Literatura. Ciência Atual-Rev Científica Multidisciplinar das Faculdades São José 2015;6:2-6. http://www.cnad.edu.br/revista-cienciaatual/index.php/cafsj/article/view/122/106

2.Cruz BMS, Martins-Filho RHG, Colaço MAXP. Reabilitação Fisioterápica De Pacientes Com Sequelas Motoras De Acidente Vascular Cerebral Isquêmico: Uma Revisão Bibliográfica. Rev Inspirar Mov Saude 2016;10:28-36.

https://www.inspirar.com.br/revista/reabilitacao-fisioterapica-depacientes-com-sequelas-motoras-de-acidente-vascular-cerebralisquemico-uma-revisao-bibliografica/

3.Abreu M, Oliveira GMR, Souza LAPS. Efeitos do fenômeno da irradiação do método de Facilitação Neuromuscular Proprioceptiva no acidente vascular encefálico sobre o membro inferior: estudo preliminar. J ConScientiae Saúde 2018;17:257-65. https://doi.org/10.5585/conssaude.v17n3.8091

4.Pola HMC, Bergmann KE, Silva JS. Desempenho Funcional em Hemiparéticos Crônicos Antes e Após Programa de Exercício com Facilitação Neuromuscular Proprioceptiva. Rev Bras Saúde Funcional 2015;2:46.

http://www.seeradventista.com.br/ojs/index.php/RBSF/article/view/663 
5. Alves NS, Paz FAN. Análise das principais sequelas observadas em pacientes vítimas de acidente vascular cerebral-AVC. Rev FAESF 2018;2:25-30.

http://faesfpi.com.br/revista/index.php/faesf/article/view/66

6. Krukowska J, Bugajski M, Sienkiewicz M, Czernicki J. The influence of NDT-Bobath and PNF methods on the field support and total path length measure foot pressure (COP) in patients after stroke. Neurol Neuroch Polska 2016;50:449-54.

https://doi.org/10.1016/j.pjnns.2016.08.004

7.Kim B-R, Kang T-W. The effects of proprioceptive neuromuscular facilitation lower-leg taping and treadmill training on mobility in patients with stroke. International journal of rehabilitation research. Internationale Zeitschrift fur Rehabilitationsforschung. Rev Inter Rech Readapt 2018;41:343-8.

https://doi.org/10.1097/MRR.0000000000000309

8. Kim CH, Kim YN. Effects of Proprioceptive Neuromuscular Facilitation and Treadmill Training on the Balance and Walking Ability of Stroke Patients. J Korean Phys Ther 2018;30:7983. https://doi.org/10.18857/jkpt.2018.30.3.79

9.Amaral DBS, Coutinho ID, Gomes NV, Ferreira KL et al. Avaliação da Funcionalidade do Membro Superior Parético de Pacientes Com Sequela de AVE Após Protocolo Terapia por Contensão Induzida. Biol Saúde 2017;7:81-7. https://doi.org/10.25242/886872420171151

10.Dutra BD. Efeitos de um tratamento apoiado na sequência ontogenética do desenvolvimento humano em hemiparéticos. (Trabalho de Conclusão do Curso). Santa Cruz do Sul: Universidade de Santa Cruz do Sul, 2015, 33p.

https://repositorio.unisc.br/jspui/handle/11624/956

11.Marchese RR. Efeito de padrões de facilitação neuromuscular proprioceptiva na irradiação motora para membro inferior contralateral (Dissertação). Porto Alegre: Universidade Federal de Ciências da saúde de Porto Alegre, 2016, 70p.

https://repositorio.ufcspa.edu.br/jspui/bitstream/123456789/447/1/ Marchese\%2C\%20Ritchele\%20Redivo Disserta\%C3\%A7\%C3\%A30.p df

12. Reis S. Intervenção fisioterapêutica em paciente acometido por AVE isquêmico. Rev Interdisc 2015;8:202-7.

https://revistainterdisciplinar.uninovafapi.edu.br/index.php/revinter/a rticle/view/486/0

13.Santos NS, Foss MHD, Ferreira LL. Facilitação neuromuscular proprioceptiva na marcha em pacientes com sequela de acidente vascular encefalico. Arq Ciênc Saúde 2016;23:87-91. https://doi.org/10.17696/2318-3691.23.2.2016.338

14.Trigueiro CQ. Funcionalidade do membro superior hemiplégico após programa de facilitação neuromuscular, liberação miofascial e kinesiotapping ®. E-RAC 2018;7.

http://www.computacao.unitri.edu.br/erac/index.php/erac/article/view/722 
15.Guiu-Tula FX, Cabanas-Valdés R, Sitjà-Rabert M, Urrutia L, Gomara-Toldrá N. The Efficacy of the proprioceptive neuromuscular facilitation (PNF) approach in stroke rehabilitation to improve basic activities of daily living and quality of life: a systematic review and meta-analysis protocol. BMJ open 2017;7:e016739. Doi: 10.1136 / bmjopen-2017-016739

16.Grant R. Upper Extremity Proprioceptive Neuromuscular Facilitation Pattern-Induced Irradiation to Improve Sitting Balance in a Stroke Patient (tese). Azusa Pacific University. 2017 https://search.proquest.com/openview/9612f56b0a3ad62b17f12531d cf97ba3/1?pq-origsite $=$ gscholar $\& \mathrm{cbl}=18750 \&$ diss $=y$

16. Melo AWS, Silva JM, Pereira TMA, Orsini M, Teixeira S, Bastos VHV. Funcionalidade e incapacidade dos pacientes pós-acidente vascular encefálico: relato de casos. Rev Pes Fisioter 2019;9:101-7. http://dx.doi.org/10.17267/2238-2704rpf.v9i1.2176 